\title{
Thinking Like a Scientist and Thinking Like a Doctor
}

\author{
Stephen Loftus ${ }^{1}$ (D) \\ Published online: 21 November 2017 \\ (C) International Association of Medical Science Educators 2017
}

Abbreviations PBL Problem-based learning

\section{Introduction}

There is a widespread belief still held among many medical practitioners and educators that the work of a doctor is much the same as the work of an applied scientist. This is a myth and one that continues to cause confusion and holds back efforts to improve both medical education and practice. This myth has a long history and is based on the unfounded assumption that diagnosing a disease and devising a treatment plan is largely identical to testing a hypothesis in a laboratory study. This commentary critically examines this myth and explores its implications. There is a need to explore ways of viewing scientific thinking and clinical reasoning that draw out the subtle, and not so subtle, differences between them and the implications for medical education. Medical education should aim to integrate the technical rationality of scientific thinking with the interpretive rationality of the humanities and social sciences. Such integration can better prepare students to master the practical rationality needed in a professional practice such as medicine [1].

\section{The Origins of the Problem}

Historically, the integration of the biomedical sciences into the practice of medicine, and medical education, has been of huge benefit. Pioneers, such as Abraham Flexner [2], realized that the biomedical sciences were needed to provide a firm foundation for medical practice and education. In North America, this resulted in medical schools being associated with centers of scientific research and an educational curriculum that

Stephen Loftus

loftus@oakland.edu

1 Oakland University William Beaumont School Of Medicine, Rochester, MI, USA insisted on rigorous scientific preparation before students could start to see patients. Over the decades since, the scientific grounding of medicine has prompted rapid advances in patient care and brought about dramatic improvements in our ability to deal with many diseases and ailments. The need for a scientific basis for medical practice is essentially beyond question. However, there seems to be some confusion about the limits of science in medicine and the need for ideas and thinking from other disciplines. For example, a critical reading of a recent AAMC document [3] asserting the importance of the biosciences in medicine reveals some of this confusion.

One claim of this document [3] is that an overarching principle for scientifically informed physicians is the need for strong ethical principles (p. 5). Ethics is not a science. Ethics concerns values and morals and is a branch of philosophy, one of the humanities. There is an attempt here to appropriate ethical thinking and pass it off as scientific thinking. Ethical thinking is an example of the interpretive rationality of the humanities. Ethics certainly needs to be integrated into the clinical reasoning of the physician but this does not make it scientific. Later, in the same document, there is a concession that medical practice can also be informed by other disciplines. Only the behavioral and social sciences are named. Presumably, the word "science" in their titles makes them acceptable. There is no explicit mention of the humanities in this document even though at least one of them (ethics) is clearly present. Scientific thinking and clinical reasoning are not the same. Conflating the two causes confusion.

The confusion is partly due to the fact that there are some similarities between scientific thinking and clinical reasoning. For example, a scientist must devise a hypothesis that can be tested by providing evidence that substantiates or refutes that hypothesis. Likewise, a doctor must devise a hypothesis about what is causing a patient's problem that should be tested. A doctor looks for evidence that a patient has, or has not, a particular diagnosis. Indeed, clinical reasoning is often seen to be the same as scientific hypothetico-deductive reasoning and this is often explicitly taught in medical schools. Problem-based learning (PBL) is one well-known example of introducing students to the hypothetico-deductive method. We often think of a 
doctor's treatment as being like a scientific experiment. If an antibiotic seems to make the patient better, then this is taken to prove the hypothesis that the patient had an infection must have been correct. However, these similarities are superficial and misleading. The similarities are emphasized but the differences are overlooked. A major difference is that different goals are driving the two activities.

\section{Different Goals}

The goals of the scientist and the doctor are not the same. A scientist is trying to establish some knowledge, some universal truth about the world we live in. In contrast, a doctor is trying to help a patient. There is a world of difference in these goals. It is true that a doctor may order a number of laboratory tests and find out scientific information about a patient, but this is more like the work of a detective rather than a scientist. It may be more useful to think of doctors as being specialized detectives or as being similar to criminal lawyers. A criminal lawyer may need to know and understand a lot of forensic science but nobody thinks of a lawyer as being a scientist. The lawyer is a scientifically informed professional practitioner who is trying to get the best result for a client. A doctor, too, is a scientifically informed professional practitioner who is trying to get the best result for a patient. The goal of medical practice and clinical reasoning is not new knowledge of the world but the best outcome for a particular patient.

In contrast, scientific thinking seeks knowledge that is timeless and universal. Scientific knowledge should be precise and reliable. It should be law-like and generalizable. Clinical reasoning seeks information that is timely and specific to this patient, in the here and now, and that will be in the best interests of this particular patient. Scientific thinking can also obscure the fact that a clinician has to deal with real people. Scientists must be objective and remain detached from their research, even if the research subjects are people. Doctors certainly need to maintain an appropriate professional distance but all too often scientific thinking has led to patients being treated impersonally and feeling that doctors do not care about them. There are already many reports of patients becoming merely the "hernia in bed six" and complaining that doctors do not treat them as human beings (e.g., [4]). Scientific thinking alone can tempt doctors to become preoccupied with biological processes and forget the real world people before them.

For example, Svenaeus [5] said:

Doctors in the clinic do not meet with agents who evaluate their pain and take a rational stand upon what they want to have done with their biological processes, but with worried, help-seeking persons, who need care and understanding ... (pp. 173-174)
There is a growing recognition that doctors need to pay much more attention to understanding the "worried, helpseeking persons" before them while not forgetting the biological processes that must still be dealt with. This extra demand to deal with the human element means that clinical reasoning can be complex in ways that are quite different to scientific thinking. Clinical reasoning is much more case-based and this is reflected in insights that medical students themselves report. For example, one student, with a strong biomedical background, realized that the scientific knowledge he had acquired over several years would need to be completely reorganized if he was to find it useful as a clinician.

"I've gone through my old notes and progressively thrown them out as I've rewritten them into a different format ... now I approach learning the diseases in the same way that I would ... a patient". ([6]; p. 100)

He was not rejecting or throwing out his scientific knowledge but reorganizing it in to a case-based format that would be more relevant to medical practice. The case-based nature of clinical reasoning means that two people with an identical biomedical diagnosis may have to be managed quite differently. For example, one patient may be a young adult who is otherwise healthy while the other patient may be a very elderly, frail person with many comorbidities. One may receive aggressive treatment and the other may get palliative care. Decisions like these are not based on scientific grounds but on what is judged to be ethically best for the patient.

The student above is one example of a common observation that many medical students struggle to relate their wealth of scientific knowledge to real patients. The students complain that they know that they have scientific knowledge "in their heads" but have difficulty working out what might be relevant for any particular patient. This is one of the reasons behind the push for more integrated curricula in medical schools. For example, in a PBL curriculum, one goal is always to work out the diagnosis and treatment for the patient for each and every case. The curriculum will usually have dozens of cases. Clinical reasoning is repeatedly emphasized and drives the learning activity. Scientific knowledge is needed but always framed in terms of how it is useful and relevant to the case and how it informs the clinical reasoning. There is scope for medical humanities scholars to have input into the design of PBL cases so that the need for integrated thinking is made explicit. A more integrated curriculum, such as one based on PBL, also tends to emphasize early patient contact so that scientific knowledge can be related to real experience with real patients. Even in a more traditional curriculum, it is important to relate scientific knowledge to clinical cases in the form of patient panels and case studies in order to help students see the links between scientific 
knowledge and clinical practice. Borrowing insights from the medical humanities and social sciences can help us understand clinical reasoning and scientific thinking in more depth.

\section{Insights from the Medical Humanities}

Montgomery [7] discussed what she called "the misdescription of medicine" (p. 29). Her point was that doctors have for a long time deceived themselves into thinking that their clinical reasoning is no more than the simplistic application of scientific thinking. Montgomery argued that doctors exercise a great deal of clinical judgment that is based on hermeneutic and narrative thinking. Hermeneutics, one of the medical humanities, is the study and practice of interpretation. Doctors must exercise a great deal of interpretive skill in clinical practice. A patient's initial presentation must be interpreted so that the doctor can devise a differential diagnosis, a list of potential causes of the patient's problem. Hermeneutics emphasizes the importance of relating parts to wholes, and back again from wholes to parts, in a circular fashion to generate a holistic understanding of a patient with a problem. To do this, doctors rely heavily on narrative, the emerging story of the patient. Narrative Medicine is yet another example of the Medical Humanities. Doctors relate the present patient's story to those of other patients with similar complaints. The other stories are usually patient cases they have directly experienced but also stories they have heard of from colleagues who can share their experience and expertise.

A key feature of expertise is not that an expert knows more science than a novice. Sometimes, the novice is more up-todate with the science than the expert. The difference is that the expert has a greater wealth of case-based (narrative) knowledge to draw on. Because of this, an expert can appreciate a subtle difference that makes a real difference. This is important because the reality of medical practice is that there is often a great deal of complexity and uncertainty, but decisions still have to be made. It is not always possible to wait and keep gathering information and doing tests until there is absolute scientific certainty. Every clinician will know the frustration of finding that the test results are not yet available or are missing or, even more frustrating, the test results are inconclusive, or ambiguous, or contradictory. Making decisions under these circumstances requires clinical judgment. Medical students are often surprised to discover just how much uncertainty there is in medicine. They often assume that because we are generating more and more scientific knowledge, we are reducing uncertainty. If anything, the opposite is true. Because we are so successful in dealing with acute problems, we have an aging population and a growing number of patients who have chronic conditions with one or more comorbidities. Uncertainty and complexity are on the rise. The move to evidence-based medicine (EBM) is an attempt to minimize complexity and uncertainty with scientific evidence but it cannot eliminate them. There is still a need for clinical judgment.

\section{EBM and Clinical Judgment}

The pioneers of EBM recognized that EBM could not replace clinical judgment but this is frequently forgotten. Sackett et al. [8] stated that the ability to make good clinical decisions was through an integration of the best available evidence together with the personal expertise and experience of the clinician, and the values and wishes of the patient. The scientific grounding of medical education and practice means that doctors have been willing and able to embrace the idea of the best available evidence but have tended to ignore the need for expertise and experience. This is probably because they are not sure how to talk about expertise or the ethical demands to take into account the values and desires of patients.

The language of laboratory science and clinical trials does not have the vocabulary to discuss expertise or ethics in any meaningful way. This is, again, where the humanities and the social sciences come to the rescue. Several humanities and social science disciplines can, and do, have the vocabularies to talk about expertise and ethics. The literature on workbased learning, for example, uses Aristotle's notion of phronesis [9], the disposition to act wisely that comes from exposure to many real world cases. Careful reflection, on the many cases experienced in real world practice, can give practitioners the insights they need to make a judgment on yet another complex and uncertain case that seems similar but is different in important ways. A danger facing medical practice and education today is the risk of narrowing down to a purely scientific worldview and assuming that scientific technical rationality is the only way to deal with the real world. This is not science but scientism.

Some in the medical profession have recognized the weakness of a biomedical approach with the call for a change to a biopsychosocial model [10]. However, the biomedical model is still dominant [11]. A more integrated view allows for the interpretive rationality of the humanities and social sciences to be included in clinical reasoning. In the wider literature on professional education, this integrated thinking has been called practical rationality [1]. This is why the medical humanities should not be a peripheral part of the medical curriculum but need to be fully integrated so that students can learn to take different perspectives on problems. Clinical judgment is the ability to integrate the clinical facts and the ethical concerns into a coherent narrative that makes sense of all the issues facing a patient.

The argument is that the social sciences can "open up for examination the diversity of human possibilities and experience" (1, p. 94), while the humanities can "provide 
means of understanding and interpreting the complexities of purpose and meaning" (p. 94). The clinical judgment needed to deal with the uncertainties and complexity of real world patient needs all these insights. It is not a question of either/ or but both/and. The question arises of how we can do this. Promising new directions include developments in narrative medicine where efforts to integrate the different modes of thinking are being made, including the ethical [4].

Sackett et al. [8] also mention the values and desires of the patient (and by implication family members). This can sometimes raise moral and ethical issues in making clinical decisions. As mentioned earlier, scientific thinking is ill-suited to decide ethical issues. The case-based interpretive rationality of the humanities is needed to work out the best possible options in an ethical decision. As Montgomery [7] pointed out, "Physicians are science-using, information-sorting interpreters of timebound circumstances." (p. 174). Ethical decisions are examples where the localized and time-bound nature of clinical reasoning is prominent. Ethical decision-making is yet another aspect of clinical judgment, the practical rationality needed in medical practice.

In summary, scientific technical rationality informs medical practice but the clinical judgment needed by doctors needs to be interdisciplinary and integrate the interpretive rationality of the medical humanities to become clinical judgment. This is the practical rationality of a professional who can deal with complexity and uncertainty. Montgomery [7] provides the insight that scientific thinking is needed to provide an answer to an EBM question but clinical judgment is needed to devise a good EBM question in the first place. We need to stop believing that clinical reasoning and judgment are the simplistic application of science and adopt an interdisciplinary approach to help us explore the complex ways in which clinicians make decisions in the real world and how we can teach this to students.
Compliance with Ethical Standards

Conflict of Interest The author declares that there is no conflict of interest.

\section{References}

1. Sullivan WM, Rosin MS. A new agenda for higher education: shaping a life of the mind for practice. San Francisco: Jossey-Bass; 2008.

2. Flexner A. Medical education in the United States and Canada. New York: Carnegie Foundation for the Advancement of Teaching; 1910.

3. AAMC-HHMI . Scientific Foundations for Future Physicians. Report of the AAMC-HHMI Committee. AAMC Washington DC; 2009.

4. Charon R, DasGupta S, Hermann N, Irvine C, Marcus ER, Rivera Colon E, et al. The principles and practice of narrative medicine. Oxford: Oxford University Press; 2017.

5. Svenaeus F. The hermeneutics of medicine and the phenomenology of health: steps towards a philosophy of medical practice. Dordrecht: Kluwer Academic; 2000. https://doi.org/10.1007/97894-015-9458-5.

6. Loftus S. Language in clinical reasoning: towards a new understanding. Saarbrucken: VDM Verlag; 2009.

7. Montgomery K. How doctors think: clinical judgment and the practice of medicine. Oxford: Oxford University Press; 2006.

8. Sackett DL, et al., editors. Evidence-based medicine: how to practice and teach EBM. New York: Churchill Livingstone; 1996.

9. Kinsella EA, Pitman A. (eds.). Phronesis as professional knowledge: practical wisdom in the professions. Sense Rotterdam: the Netherlands; 2012.

10. Engel GL. The need for a new medical model: a challenge for biomedicine. Science. 1977;196(4286):129-36. https://doi.org/10. 1126/science. 847460.

11. Wade DT, Halligan PW. The biopsychosocial model of illness: a model whose time has come. Clin Rehabil. 2017;31(8):995-1004. https://doi.org/10.1177/0269215517709890. 